\section{Disclosures}

No Conflicts of interest to disclose.

\section{Contributions}

None.

\section{References}

1. Beutler E. Energy metabolism and maintenance of erythrocytes. In: Williams WJ, Beutler E, Erslev A, Lichtman M, editors. Hematology. 4th ed. New York, NY, USA: McGraw-Hill; 1990. pp. 355-368.

2. Brown KA. Erythrocytes metabolism and defects. Lab Med. 1996;27(5):329-333.

3. Beutler E, Gelbart T. Estimating the prevalence of pyruvate kinase deficiency from the gene frequency in the general white population. Blood. 2000;95(11):3585-3588.

4. Zanella A, Fermo E, Bianchi P, Valentini G. Red cell pyruvate kinase deficiency: molecular and clinical aspects. $\mathrm{Br} \mathrm{J}$ Haematol. 2005;130(1):11-25.

5. Jurica MS, Mesecar A, Heath PJ, Shi W, Nowak T, Stoddard BL. The allosteric regulation of pyruvate kinase by fructose-1,6-bisphosphate. Structure. 1998;6(2):195-210.

6. Kung C, Hixon J, Kosinski PA, et al. AG-348 enhances pyruvate kinase activity in red blood cells from patients with pyruvate kinase deficiency. Blood. 2017;130(11):1347-1356.

7. Perutz MF. Stereochemistry of cooperative effects in haemoglobin. Nature. 1970;228(5273):726-739.

8. Rab MAE, van Oirschot BA, Kosinski PA, et al. AG-348 (Mitapivat), an allosteric activator of red blood cell pyruvate kinase, increases enzymatic activity, protein stability, and ATP levels over a broad range of PKLR genotypes. Haematologica. 2020;106(1):238-249.

9. Rogers, SC, Ross JGC, d'Avignon A, et al. Sickle hemoglobin disturbs normal coupling among erythrocyte $\mathrm{O} 2$ content, glycolysis, and antioxidant capacity. Blood. 2013;121(9):1651-1662.

10. Grace RF, Rose C, Layton M, et al. Safety and efficacy of mitapivat in pyruvate pinase deficiency. N Engl J Med. 2019;381(10):933-944.

11. Delivoria-Papadopoulos M, Oski F, Gottlib AJ. Oxygen-hemoglobin dissociation curves: effect of inherited enzyme defects in the red cell. Science. 1969;165(3893):601-602.

12. Jana S, Strader MB, Meng F, Hicks W, et al. Hemoglobin oxidationdependent reactions promote interactions with band 3 and oxidative changes in sickle cell-derived microparticles. JCI Insight. 2018;3(21): e120451.

\title{
The arrival of personalized genomics in bone marrow failure
}

\section{Marcin W. Wlodarski}

Department of Hematology, St. Jude Children's Research Hospital, Memphis, TN, USA and Division of Pediatric Hematology and Oncology, Department of Pediatrics and Adolescent Medicine, Medical Center, Faculty of Medicine, University of Freiburg, Freiburg, Germany

E-mail: MARCIN WWLODARSKI - marcin.wlodarski@stjude.org

doi:10.3324/haematol.2020.265124

F rom the description of a first genetic bone marrow failure (BMF) syndrome (dyskeratosis congenita) in the literature ${ }^{1}$ it took over 80 years to discover FANCA as the first gene causing BMF in humans. ${ }^{2}$ Using a laborious process of positional cloning, which starts by finding patients with similar phenotype patterns, performing linkage analysis using polymorphic markers to finally localize the affected cDNA, a number of additional BMF genes have been identified in the following years. These include DKC1 discovered in 1998, ${ }^{3}$ RPS19 in $1999^{4}$ and ELANE in $1999,{ }^{5}$ to name just a few. Fueled by mapping the human genome and rapid advances in genome-wide technologies, a large proportion of genes predisposing to BMF and myelodysplastic syndromes (MDS) were identified using next-generation sequencing (NGS) technologies in the $21^{\text {st }}$ century. Overall, roughly 100 genes have, thus, so far been associated with BMF/MDS syndromes. Classical inherited BMF syndromes can be categorized into DiamondBlackfan anemia (DBA), Fanconi anemia (FA), severe congenital neutropenia (SCN), dyskeratosis congenita (DC), Shwachman-Diamond syndrome, and congenital thrombocytopenias (Figure 1A). From a biological perspective, all these entities are caused by loss of function of fundamental cellular pathways such as DNA repair, ribosome or telomere maintenance. In recent years we have witnessed a series of new discoveries on hereditary conditions predisposing to MDS. ${ }^{6-8}$ The majority of these MDS predisposing genes are essential hematopoietic transcription factors and result in heterogeneous phenotypes that can bridge over to the classical BMF spectrum.

The landscape of germline genetic changes in BMF/MDS syndromes includes mutations in coding regions or noncoding alterations in promoters, regulatory elements, synonymous mutations, small deletions spanning single exons, and whole gene deletions (Figure 1B). Somatic alterations are mostly point mutations in leukemia driver genes and chromosomal gains or losses, but revertant uniparental disomies are also frequently found. Most BMF experts perform a phenotype-driven approach by first obtaining a family and medical history, followed by detailed physical examination and analysis of blood counts and bone marrow cellularity, morphology and cytogenetics. This approach most often guides the selection of an appropriate genetic testing platform to identify the causative gene (Figure 1C). For example, deletions (whole gene or intragenic) are a common cause of DBA and FA and for their detection, a well-established clinical copy number method is required (such as high resolution comparative genomic hybridization/single nucleotide polymorphism [CGH/SNP] array or multiplex ligation-dependent probe amplification [MLPA]). On the other hand, intronic noncoding mutations in GATA2 might escape standard whole exome sequencing (WES) diagnostics because of coverage gaps, or simply due to missing expert knowledge about such lesions. The diagnostic genetic process for BMF and hereditary $\mathrm{MDS}$ is often challenging due to the continuous addition of novel genotypes and the evolving phenotype spectrum, but also the clinical need for a rapid turnaround 


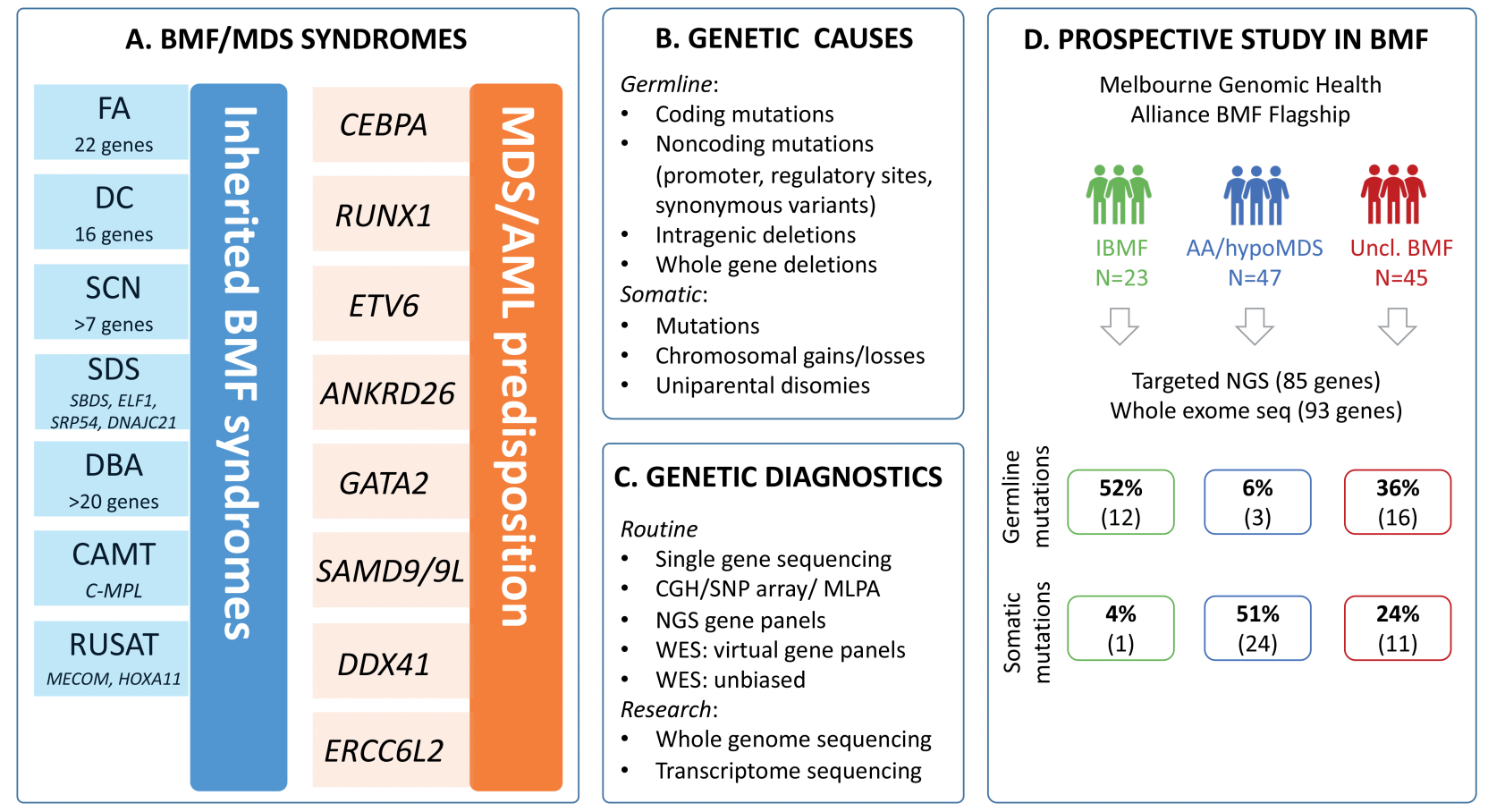

Figure 1. Genomics of bone marrow failure and hereditary myelodysplastic syndromes. (A) Syndromes and associated genes. (B) Genetic changes encountered in bone marrow failure/ hereditary myelodysplastic syndromes (BMF/MDS). (C) Genetic methods required to identify causative genetics. (D) Outline of study by Blombery et al. 2020. FA: Fanconi anemia; DC: dyskeratosis congenita; SCN: severe congenital neutropenia; SDS: Shwachman-Diamond syndrome; DBA: Diamond Blackfan anemia; CAMT: congenital amegakaryocytic thrombocytopenia; RUSAT: radioulnar synostosis with amegakaryocytic thrombocytopenia; IBMF: inherited BMF; AA/hypoMDS: aplastic anemia/hypocellular MDS; Uncl. BMF: unclassified BMF; CGH: comparative genomic hybridization; SNP: single nucleotide polymorphism; MLPA: multiplex ligation-dependent probe amplification; NGS: next-generation sequencing; WGS: whole exome sequencing.

time to guide therapeutic decisions.

Blombery and co-workers ${ }^{6}$ are now presenting a very innovative multi-institutional prospective study from the Melbourne Genomic Health Alliance BMF Flagship (Figure 1D). They analyzed a series of 115 pediatric and adult patients diagnosed consecutively with BMF at four institutions in Australia between May 2017 and August 2018. The main purpose was to assess the impact of comprehensive genomic evaluation in this heterogenous cohort. The authors performed a tour de force analysis using a genomic pipeline consisting of WES, targeted NGS, and in selected cases additional RNA sequencing (RNAseq) to confirm variant effect on RNA expression and droplet digital PCR (ddPCR) technique to validate small somatic clones. Every patient received personalized genetic counseling and the pathogenic variants identified through this study were used for clinical decision making. Before starting genetic analysis, patients were grouped into three diagnostic categories: inherited BMF (23 patients), acquired aplastic anemia (AA)/hypocellular MDS (47 patients) and unclassified BMF (45). The analysis resulted in a change of diagnostic category in $26 \%$ of the cohort. Most notably, the authors discovered germline causes in 3 of 47 and 16 of 45 of patients with pretest diagnoses of AA/MDS and clinically unclassifiable BMF, respectively. Additionally, somatic mutations were discovered in $51 \%$ of $\mathrm{AA} / \mathrm{MDS}$ group, $24 \%$ of unclassified BMF, and $4 \%$ of inherited BMF (Figure 1D).

Recent studies have demonstrated the utility of comprehensive genomic analysis in diagnosing BMF and hereditary MDS. Zhang and colleagues employed an NGS panel of 85 genes to find that 8 of 71 patients with idiopathic $\mathrm{BMF} / \mathrm{MDS}$ carry damaging germline mutations in GATA2, RUNX1, DKC1 or LIGIV. ${ }^{9}$ They also demonstrated the utility of NGS panels to detect genomic copy variants in BMF. Another study focused on finding copy number variants in a large cohort consisting of BMF patients and found that $16 \%$ of them carry pathogenic copy number variants. ${ }^{10}$ A landmark study by a French group in 2018 investigated 179 patients with suspected but genetically unresolved BMF. Using WES sequencing of fibroblast DNA, they found pathogenic mutations affecting 28 genes in $48 \%$ of this cohort. ${ }^{11}$ Finally, in a most recent study using WES performed in 86 families with MDS/AML, known genes were found mutated in $57 \%$ of families, and 65 new candidate loci were discovered in $43 \%$ of cases. ${ }^{12}$ An important finding of this work was that there is no single gene that accounts for a large number of unresolved cases with familial disease. However, aforementioned studies were of retrospective nature in cohorts collected over many years to decades. This leads to several questions: What is the immediate benefit to patient care? Should investigators approach families about pathogenic variants found in research only studies and if yes, how do we do this?

In the current study, Blombery et al. ${ }^{13}$ used an accredited genomic pipeline where results can be returned immediately after testing to clinicians and patients. This approach had important implications for clinical management, including the choice to perform allogeneic stem cell transplantation and optimal sibling donor selection, treatment (such as corticosteroids in DBA), identification of at risk family members and implementation of disease-specific 
surveillance. Several examples showcased the importance of such a real-time prospective study for patients with BMF. In one family, the identification of a pathogenic TINF2 mutation permitted family screening and implementing DC-specific surveillance. In another family, diagnosis of homozygous $R A D 51 C$ Fanconi-like syndrome had implications for breast and ovarian cancer screening in family members. Interestingly, in one case with a newly identified homozygous FANCA mutation (that resulted in exon skipping based on RNAseq), the chromosomal fragility test was ambiguous. This emphasizes the clinical impacts of personalized genomics in BMF patients, especially when the clinical features and functional testing do not completely align with a BMF syndrome, to obtain an accurate diagnosis and avoid missed or delayed diagnosis.

NGS technologies have already fulfilled their promise as a diagnostic tool in BMF/MDS. Now, we should ride on this impressive momentum and take advantage of international consortia to implement a rapid diagnostic pipeline for these rare disorders in a prospective setting, as has already been demonstrated for pediatric cancers and seriously ill infants. ${ }^{14,15}$ The virtue will be to use rapid "all-inone" genomic platforms such as WGS complemented by RNAseq allowing simultaneous detection of all types of disease-relevant genomic lesions. Personalized genomics for patients with BMF has finally arrived.

\section{Disclosures}

No Conflicts of interest to disclose.

\section{Contributions}

None.

\section{References}

1.Zinsser F. Atrophia cutis reticularis cum pigmentatione dystrophia unguium et leukoplakia oris. Ikonographia Dermatologica. 1906 1906;5:219-223.

2. Strathdee CA, Gavish H, Shannon WR, Buchwald M. Cloning of cDNAs for Fanconi's anaemia by functional complementation. Nature. 1992;356(6372):763-767.

3. Heiss NS, Knight SW, Vulliamy TJ, et al. X-linked dyskeratosis congenita is caused by mutations in a highly conserved gene with putative nucleolar functions. Nat Genet. 1998;19(1):32-38.

4. Draptchinskaia N, Gustavsson P, Andersson B, et al. The gene encoding ribosomal protein S19 is mutated in Diamond-Blackfan anaemia. Nat Genet. 1999;21(2):169-175.

5. Horwitz M, Benson KF, Person RE, Aprikyan AG, Dale DC Mutations in ELA2, encoding neutrophil elastase, define a 21-day biological clock in cyclic haematopoiesis. Nat Genet. 1999;23(4):433436.

6. Khincha PP, Savage SA. Genomic characterization of the inherited bone marrow failure syndromes. Semin Hematol. 2013;50(4):333347.

7. Kennedy AL, Shimamura A. Genetic predisposition to MDS: clinical features and clonal evolution. Blood. 2019;133(10):1071-1085.

8. Sahoo SS, Kozyra EJ, Wlodarski MW. Germline predisposition in myeloid neoplasms: Unique genetic and clinical features of GATA2 deficiency and SAMD9/SAMD9L syndromes. Best Pract Res Clin Haematol. 2020;33(3):101197.

9. Zhang MY, Keel SB, Walsh T, et al. Genomic analysis of bone marrow failure and myelodysplastic syndromes reveals phenotypic and diagnostic complexity. Haematologica. 2015;100(1):42-48.

10. Waespe N, Dhanraj S, Wahala M, et al. The clinical impact of copy number variants in inherited bone marrow failure syndromes. NPJ Genom Med. 2017;2:18.

11. Bluteau $O$, Sebert M, Leblanc T, et al. A landscape of germ line mutations in a cohort of inherited bone marrow failure patients. Blood. 2018;131(7):717-732

12. Rio-Machin A, Vulliamy T, Hug N, et al. The complex genetic landscape of familial MDS and AML reveals pathogenic germline variants. Nat Commun. 2020:11(1):1044

13. Blombery P, Fox L, Ryland GL, et al. Utility of clinical comprehensive genomic characterisation for diagnostic categorisation in patients presenting with hypocellular bone marrow failure syndromes. Haematologica. 2021;106(1):64-73

14. Rusch M, Nakitandwe J, Shurtleff S, et al. Clinical cancer genomic profiling by three-platform sequencing of whole genome, whole exome and transcriptome. Nat Commun. 2018;9(1):3962.

15. Clark MM, Hildreth A, Batalov S, et al. Diagnosis of genetic diseases in seriously ill children by rapid whole-genome sequencing and automated phenotyping and interpretation. Sci Transl Med. 2019;11(489):eaat6177. 